\title{
Mode-matching analysis and design of substrate integrated waveguide T-junction diplexer and corner filter
}

\author{
Zamzam Kordiboroujeni* ${ }^{\dagger}$ and Jens Bornemann \\ Department of Electrical and Computer Engineering, University of Victoria, Victoria, BC, V8W 2Y2 Canada
}

\begin{abstract}
SUMMARY
The mode-matching technique is deployed in this paper in order to analyze and design a substrate integrated waveguide (SIW) T-junction diplexer in Ku-band. The diplexer has bandwidths of $4.12 \%$ and $3.79 \%$ at 14.55 and $15.85 \mathrm{GHz}$, respectively. Also in Ku-band, an SIW corner filter is analyzed and designed with the same technique. Analytical results are compared with simulated data obtained from commercially available field solvers such as CST Microwave Studio and ANSYS HFSS. Excellent agreement between analytical and simulated data is achieved. Copyright @ 2014 John Wiley \& Sons, Ltd.
\end{abstract}

Received 6 May 2014; Revised 4 September 2014; Accepted 9 September 2014

KEY WORDS: substrate integrated waveguide; mode-matching techniques; diplexer; filter; T-junction; corner.

\section{INTRODUCTION}

The advent of substrate integrated circuit technology, and specifically substrate integrated waveguide (SIW) technology, has introduced a new layout to efficiently design microwave and millimeter-wave structures. Integrable with other planar topologies, these compact and high Q-factor structures can easily be fabricated at a low cost. Numerous efforts in the area of design and analysis of passive SIW components, including diplexers, have been undertaken in the past few years.

One of the essential passive components in front-end systems is the diplexer, which provides needed separation between transmit and receive bands. The first diplexers based on SIW technology are reported in 2005 [1,2]. An X-band SIW diplexer using asymmetric dual-mode iris filters in a branchingport configuration is proposed in [1]. The bands of the diplexer are located at 9.7 and $10.3 \mathrm{GHz}$, respectively, and three-pole in-band filters are used at each branch [1]. A similar configuration with single-mode iris filters operates at C-band [2]. The bands of this diplexer are located at 5.42 and $5.96 \mathrm{GHz}$ using five-pole and four-pole channel filters, respectively [2]. In 2007, a diplexer designed with triplet technology is presented [3]. The diplexer has input and output ports on opposite sides of the substrate and uses iris filters, including a trisection, at 12.1 and $11.55 \mathrm{GHz}$, respectively [3]. Also in 2007, a diplexer operating at K-band uses dual mode SIW filters with circular and elliptic cavities [4]. The diplexer bands are located at 25 and $26 \mathrm{GHz}$, respectively [4]. An SIW triplexer with bands located at 1.9, 2.018, and $2.35 \mathrm{GHz}$ is reported in 2009 [5], but the insertion loss in one of the channels is in the order of $7 \mathrm{~dB}$. In 2011, a T-junction diplexer with iris filters is presented in [6] with bands located at 59.8 and $62.2 \mathrm{GHz}$, respectively; however, the performance is not verified by measurements. Also in 2011, the T-junction SIW diplexer in [7] uses complementary split-ring resonators at C-band. The channels of this C-band diplexer are located at 4.7 and $5.8 \mathrm{GHz}$. In 2013, a K-band SIW diplexer with bands located at 18.15 and $19 \mathrm{GHz}$ is introduced [8], which has been designed using an efficient

\footnotetext{
${ }^{*}$ Correspondence to: Zamzam Kordiboroujeni, Department of Electrical and Computer Engineering, University of Victoria, Victoria, BC, V8W 2Y2, Canada.

${ }^{\dagger}$ E-mail: zkordi@ece.uvic.ca
} 
mode-matching technique (MMT) proposed for SIW structures $[9,10]$. The SIW diplexer is similar in configuration to [3] and [5], but operates at a significantly higher frequency.

In all SIW diplexers mentioned earlier, except for the one reported in [8], the optimization and tuning steps of the SIW diplexer design are carried out using full-wave simulators. This is a tedious and cumbersome task, considering the large number of optimization parameters present in SIW diplexer designs. In contrast, the MMT approach adopted in [8] for designing SIW diplexers is an order of magnitude faster than commercially available field solvers. However, the presented MMT analysis cannot be directly applied for the analysis of SIW structures including T-junctions and corners.

In this paper, the MMT approach presented in $[9,10]$, in conjunction with a new approach for deploying MMT in analyzing SIW T-junctions, is adopted in order to design and analyze SIW T-junction structures, such as an SIW T-junction diplexer operating at Ku-band. The diplexer bands are placed at 14.55 and $15.85 \mathrm{GHz}$, using five-pole and four-pole filters, respectively. After presenting the analytical method for SIW T-junction structures, as the next step, one of the branches in the T-junction is short circuited, and SIW corners are investigated. An SIW corner filter at Ku-band is designed based on the proposed method.

\section{THEORY}

Substrate integrated waveguide structures are planar realizations of conventional rectangular waveguides. As a result, the field patterns inside these structures are similar. However, as there is only a reduced set of modes in SIW structures [11], that is, only $T E_{m 0}$ modes, the modal analysis techniques, such as the MMT, are a proper choice for the analysis of these structures. This section starts with an overview of the MMT analysis of SIW structures and is followed by the proposed technique for analyzing SIW T-junctions.

\subsection{Mode-matching analysis of SIW structures}

Figure 1(a) shows the SIW topology with its structural parameters. On a specific dielectric with $h$ as the substrate height and $\epsilon_{r}$ as the substrate relative permittivity, the main structural parameters of an SIW structure are the diameter of the metal posts $(d)$, the space between them in a row (via pitch $p$ ), and the SIW width, $a_{S I W}$. In the design of SIW structures, the ratio of the via diameter to the via pitch, $\frac{d}{p}$, is chosen so that the electromagnetic field is completely bound inside the structure and minimum leakage occurs $(0.5<d / p<0.8$ [12]). As a result of that, we bound the SIW structure inside a larger rectangular waveguide with the same height as the SIW structure (Figure 1(b)), in order to apply the MMT.

Inside a waveguide with electric walls (E.W.), for the $T E_{m 0}$ set of modes, the magnetic vector potential can be expressed as (suppose that the cross section of the structure is in $x-y$ plane, cf. Figure 1(a)):

$$
\vec{A}_{h}=A_{h z} \vec{a}_{z}=\sum_{m} \sqrt{Z_{h m}} T_{h m}(x)\left[F_{m} e^{-j k_{z m} \cdot z}+B_{m} e^{+j k_{z m} \cdot z}\right]
$$

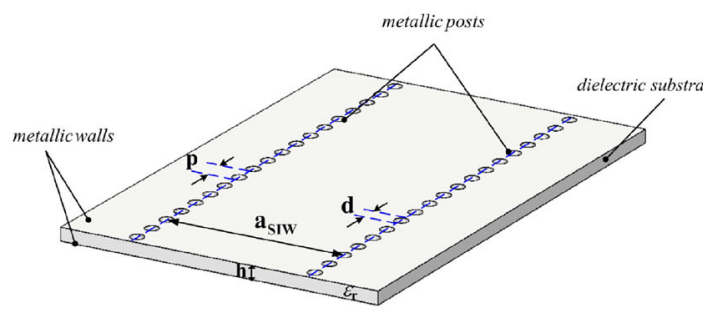

(a)

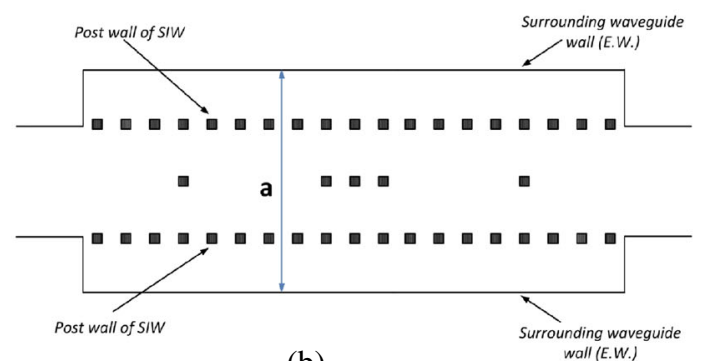

(b)

Figure 1. (a) SIW circuit with its structural parameters; and (b) a typical SIW structure with square vias surrounded by a larger rectangular waveguide. 


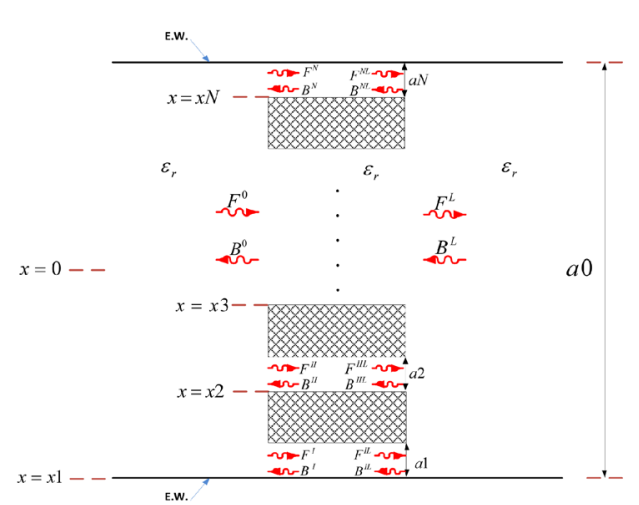

(a)

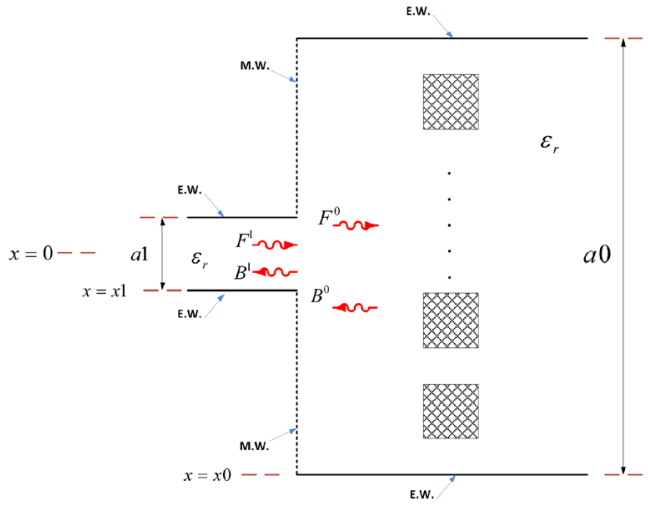

(b)

Figure 2. (a) Discontinuities between an all-dielectric waveguide and an $\mathrm{N}$-furcated waveguide formed by $N-1$ via holes; and (b) discontinuity between a waveguide port and an SIW structure.

in which $Z_{h m}$ is the wave impedance of the $T E_{m 0}$ mode and is equal to:

$$
\sqrt{Z_{h m}}=\sqrt{\frac{\omega \mu}{k_{z m}}}
$$

$\omega$ is the angular frequency, $\mu$ is the permeability of free space, $a$ is the width of the waveguide, and $k_{z m}$ is the wave propagation constant for the $T E_{m 0}$ wave in the $z$-direction and is given by:

$$
k_{z m}=\sqrt{\left(\frac{\omega}{v_{c}}\right)^{2} \epsilon_{r}-\left(\frac{m \pi}{a}\right)^{2}}
$$

in which $v_{c}$ is the speed of light in free space. $F$ and $B$ are the amplitudes of forward and backward traveling waves in the structure (see Figure 2(a)), and $T_{h}$ represents the dependence of the potential on cross-sectional coordinates; which for $T E_{m 0}$ modes, we only have an $x$ dependence of $T_{h} . T_{h}$ can be expressed as (note that the origin has been placed in the middle of the cross section):

$$
T_{h m}(x)=A_{m} \cos \frac{m \pi}{a}\left(x+\frac{a}{2}\right)
$$

Because in MMT all modes have to be normalized to the same power, for example, $1 \mathrm{~W}$ in each region, we have:

$$
A_{m}=\frac{a}{m \pi} \sqrt{\frac{2}{a h}}
$$

For the electric and magnetic fields in this region, we have:

$$
\vec{E}=-\nabla \times\left(A_{h z} \hat{a_{z}}\right) \Longrightarrow E_{y}=\frac{\partial A_{h z}}{\partial x}
$$

and

$$
\vec{H}=\frac{-1}{j \omega \mu} \frac{\partial^{2} A_{h z}}{\partial x \partial z} \hat{a_{x}}+\frac{j}{\omega \mu} \frac{\partial^{2} A_{h z}}{\partial x^{2}} \hat{a_{z}}
$$

For completing the MMT, subject to the boundary conditions at the discontinuity, electric and magnetic fields at both sides of a discontinuity relate to each other and form the S-parameters of the junction. 
2.1.1. Discontinuity between an all-dielectric waveguide and an $N$-furcated waveguide. Figure 2(a) presents the discontinuity between an all-dielectric waveguide and an $\mathrm{N}$-furcated waveguide with $N-1$ via holes. Please note that we apply the MMT for the analysis of SIW structures with rectangular/square vias. The size of the vias in Figure 2(a) is exaggerated in order to demonstrate forward and backward traveling waves in each region.

For this type of discontinuity, $\vec{E}$ and $\vec{H}$ are calculated by Equations (6) and (7), and at the discontinuity, the boundary condition of perfect electric conducting vias should be applied. By matching $E_{y}$ over the entire discontinuity (suppose that it is placed at $z=0$ ), we have:

$$
\begin{aligned}
& \sum_{m} \sqrt{\frac{\omega \mu}{k_{z m}^{0}}} A_{m}^{0} \frac{m \pi}{a_{0}}\left(-\sin \frac{m \pi}{a_{0}}\left(x+\frac{a_{0}}{2}\right)\right)\left[F_{m}^{0}+B_{m}^{0}\right] \\
= & \sum_{N} \sum_{k} \sqrt{\frac{\omega \mu}{k_{z k}^{N}}} A_{k}^{N} \frac{k \pi}{a_{N}}\left(-\sin \frac{k \pi}{a_{N}}\left(x-x_{N}\right)\right)\left[F_{k}^{N}+B_{k}^{N}\right]
\end{aligned}
$$

and by matching $H_{x}$ in each individual region, we obtain:

$$
\begin{aligned}
& \sum_{m} \frac{k_{z m}^{0}}{\omega \mu} \sqrt{\frac{\omega \mu}{k_{z m}^{0}}} A_{m}^{0} \frac{m \pi}{a_{0}}\left(-\sin \frac{m \pi}{a_{0}}\left(x+\frac{a_{0}}{2}\right)\right)\left[-F_{m}^{0}+B_{m}^{0}\right] \\
= & \sum_{k} \frac{k_{z k}^{N}}{\omega \mu} \sqrt{\frac{\omega \mu}{k_{z k}^{N}}} A_{k}^{N} \frac{k \pi}{a_{N}}\left(-\sin \frac{k \pi}{a_{N}}\left(x-x_{N}\right)\right)\left[-F_{k}^{N}+B_{k}^{N}\right]
\end{aligned}
$$

We multiply both sides of Equation (8) by $\sin \frac{m^{\prime} \pi}{a_{0}}\left(x+\frac{a_{0}}{2}\right)$ and integrate from $-\frac{a_{0}}{2}$ to $\frac{a_{0}}{2}$. This results in the following equation:

$$
F^{0}+B^{0}=\sum_{N} M^{N}\left[F^{N}+B^{N}\right]
$$

or in matrix format:

$$
\left[F^{0}+B^{0}\right]=\left[\begin{array}{llll}
M^{1} & M^{2} & \cdots & M^{N}
\end{array}\right]\left[\begin{array}{c}
F^{1}+B^{1} \\
\cdots \\
\vdots \\
\cdots \\
F^{N}+B^{N}
\end{array}\right]
$$

in which $M=\left[M^{1} M^{2} \cdots M^{N}\right]$. Also, we multiply both sides of Equation (9) by $\sin \frac{k^{\prime} \pi}{a_{N}}\left(x-x_{N}\right)$ and integrate from $x_{N}$ to $x_{N}+a_{N}$, leading to the following equation:

$$
\left(M^{N}\right)^{T}\left[F^{0}-B^{0}\right]=F^{N}-B^{N} \quad(N-\text { times })
$$

or in matrix format:

$$
\left[\begin{array}{c}
\left(M^{1}\right)^{T} \\
\cdots \\
\left(M^{2}\right)^{T} \\
\cdots \\
\vdots \\
\cdots \\
\left(M^{N}\right)^{T}
\end{array}\right] \cdot\left[F^{0}-B^{0}\right]=\left[\begin{array}{c}
F^{1}-B^{1} \\
\cdots \\
\vdots \\
\cdots \\
F^{N}-B^{N}
\end{array}\right]
$$


$M^{T}$ is the transpose of $M$. In the aforementioned equations, matrix $M^{N}$ is related to the impedance matrix $Z_{K}^{N}$, admittance matrix $Y_{m}^{0}$, and coupling integral $\left(J^{N}\right)_{m k}$ by:

$$
M^{N}=\operatorname{Diag} \sqrt{Y_{m}^{0}} \cdot J^{N} \cdot \operatorname{Diag} \sqrt{Z_{k}^{N}}
$$

and the coupling integral is:

$$
\left(J^{N}\right)_{m k}=\frac{2}{\sqrt{a_{0} a_{N}}} \int_{x_{N}}^{x_{N}+a_{N}} \sin \frac{m \pi}{a_{0}}\left(x+\frac{a_{0}}{2}\right) \sin \frac{k \pi}{a_{N}}\left(x-x_{N}\right) d x
$$

From this $M$ matrix, the S-parameters, which are relating incident and reflected wave amplitudes as:

$$
\left[\begin{array}{c}
B^{0} \\
F^{I} \\
\vdots \\
F^{N}
\end{array}\right]=[S]\left[\begin{array}{c}
F^{0} \\
B^{I} \\
\vdots \\
B^{N}
\end{array}\right]
$$

can be derived as:

$$
\begin{aligned}
& S_{11}=\left[M M^{T}+I\right]^{-1} \cdot\left[M M^{T}-I\right] \\
& S_{12}=2\left[M M^{T}+I\right]^{-1} \cdot M=S_{21}^{T} \\
& S_{21}=M^{T}\left(I-S_{11}\right) \\
& S_{22}=I-M^{T} S_{12}
\end{aligned}
$$

where $I$ denotes the unit matrix.

The maximum number of modes in each individual section is set by a maximum frequency. With this maximum frequency, $f_{\max }$, the number of modes in a waveguide section with width $a_{N}$ is determined as:

$$
m_{\max }=\text { ceiling }\left(\frac{2 a_{N} f_{\max }}{v_{p}}\right)
$$

in which $v_{p}$ is the wave propagation speed in the dielectric, and the ceiling function maps a real number to the smallest following integer.

According to our investigations, setting this $f_{\max }$ equal to 40 times the cutoff frequency of the input/output ports, $f_{c}$, results in an acceptable accuracy. $f_{c}$ is specified at the beginning of the design and determines the operation frequency band.

2.1.2. Discontinuity between waveguide ports and an SIW structure. Figure 2(b) represents the discontinuity from a waveguide port to an SIW structure. The boundary between two structures is treated as a perfect magnetic conductor (PMC). In this case, $\vec{E}$ and $\vec{H}$ are calculated by Equations (6) and (7). However, as the boundary condition is a PMC, for matching, we have to multiply both sides of the equation for $H_{x}$ by $\sin \frac{m^{\prime} \pi}{a_{0}}\left(x+\frac{a_{0}}{2}\right)$ and integrate from $-\frac{a_{0}}{2}$ to $\frac{a_{0}}{2}$ (see Figure 2(b)) and for $E_{y}$ by $\sin \frac{k^{\prime} \pi}{a_{1}}\left(x+\frac{a_{1}}{2}\right)$ and integrate from $-\frac{a_{1}}{2}$ to $\frac{a_{1}}{2}$. In this case, the coupling integral is:

$$
(J)_{m k}=\frac{2}{\sqrt{a_{0} a_{1}}} \int_{-\frac{a_{1}}{2}}^{\frac{a_{1}}{2}} \sin \frac{m \pi}{a_{0}}\left(x+\frac{a_{0}}{2}\right) \sin \frac{k \pi}{a_{1}}\left(x+\frac{a_{1}}{2}\right) d x
$$

The $M$ matrix and S-parameters are calculated as in Equations (12) and (14). 
2.1.3. MMT analysis of multiport SIW structures. If there is more than one waveguide port at the input or output of the structure, the procedure of calculating the $S$ matrix is predominantly based on the method presented earlier for two-port SIW structures. After calculation of the total modal $S$ matrix of the discontinuities involved (same procedure as that presented for two-port structures), the desired S-parameters of the multiple port structure ( $M+N$ port structure) with $M$ input ports and $N$ output ports, $S_{i j}$, are extracted from the computed overall modal two-port scattering matrix as:

$$
\begin{aligned}
S^{\text {total }}= & =\left[\begin{array}{cccccc}
S_{11}^{2-\text { port }} & S_{12}^{2-\text { port }} \\
\hdashline S_{21}^{2-\text { port }} & S_{22}^{2-\text { port }}
\end{array}\right] \\
= & {\left[\begin{array}{cccccc}
S_{11} & \cdots & S_{1 M} & S_{1(M+1)} & \cdots & S_{1(M+N)} \\
S_{21} & \cdots & S_{2 M} & S_{2(M+1)} & \cdots & S_{2(M+N)} \\
\vdots & \ddots & \vdots & \vdots & \ddots & \vdots \\
S_{M 1} & \cdots & S_{M M} & S_{M(M+1)} & \cdots & S_{M(M+N)} \\
\hdashline S_{(M+1) 1} & \cdots & S_{(M+1) M} & S_{(M+1)(M+1)} & \cdots & S_{(M+1)(M+N)} \\
\vdots & \ddots & \vdots & \vdots & \ddots & \vdots \\
S_{(M+N) 1} & \cdots & S_{(M+N) M} & S_{(M+N)(M+1)} & \cdots & S_{(M+N)(M+N)}
\end{array}\right] }
\end{aligned}
$$

\subsection{MMT for SIW T-junctions}

Mode-matching technique analysis of a waveguide T-junction is previously reported in the literature. For example, it is suggested in [13] that three short circuits with different lengths be used to terminate each port three times, and then an MMT approach is deployed. However, this approach computes the Sparameters of the fundamental mode only. The generalized modal scattering matrix for mode-matching problems involving discontinuity-distorted multi-port junctions is presented in [14]. However, the presented method requires very complex computations. In this section, an efficient MMT approach for the analysis of SIW T-junctions is presented.

The MMT analysis presented in subsection 2.1. cannot directly be deployed for the analysis of SIW T-junctions. For the S-parameters calculations of the SIW T-junction (Figure 3(c)), we first add a 'waveguide corner' (Figure 3(b)) to this T-junction and then calculate the S-parameters of this new SIW structure with one input port and two output ports (Figure 3(a)) from the theory presented in Section 2.1. Now, we subtract the S-parameters of the waveguide corner [15] from that calculated S-parameters in order to achieve the desired S-parameter of the SIW T-junction in Figure 3(c) [16]. The

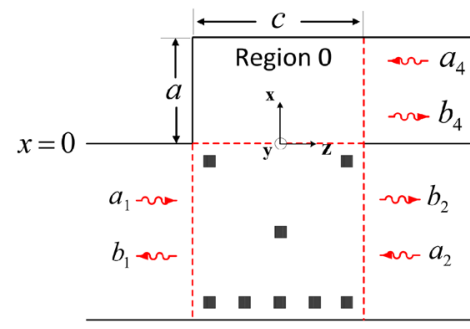

(a) I

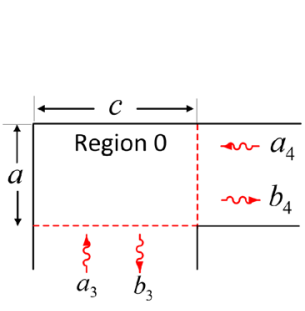

(b) II

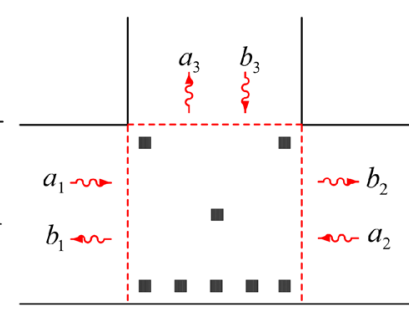

(c) III

Figure 3. S-parameter calculation of an SIW T-junction (c) based on subtraction of the S-parameters of the waveguide corner (b) from the S-parameters of an SIW structure with one input port and two output ports (a). 
final S-parameters of the SIW T-junction (structure (III)) in terms of the S-parameters of the structures (I) and (II) are as follows:

$$
\begin{aligned}
& S_{11}^{I I I}=S_{11}^{I}-S_{14}^{I} W\left(S_{44}^{I}-S_{44}^{I I}\right)^{-1} S_{41}^{I} \\
& S_{12}^{I I I}=S_{12}^{I}-S_{14}^{I} W\left(S_{44}^{I}-S_{44}^{I I}\right)^{-1} S_{42}^{I}=\left(S_{21}^{I I}\right)^{T} \\
& S_{13}^{I I I}=S_{14}^{I} W\left(S_{44}^{I}-S_{44}^{I I}\right)^{-1} S_{43}^{I I}\left(S_{33}^{I I}\right)^{-1}=\left(S_{31}^{I I I}\right)^{T} \\
& S_{21}^{I I I}=S_{21}^{I}-S_{24}^{I} W\left(S_{44}^{I}-S_{44}^{I I}\right)^{-1} S_{41}^{I}=\left(S_{12}^{I I I}\right)^{T} \\
& S_{22}^{I I I}=S_{22}^{I}-S_{24}^{I} W\left(S_{44}^{I}-S_{44}^{I I}\right)^{-1} S_{42}^{I} \\
& S_{23}^{I I I}=S_{24}^{I} W\left(S_{44}^{I}-S_{44}^{I I}\right)^{-1} S_{43}^{I I}\left(S_{33}^{I I}\right)^{-1}=\left(S_{32}^{I I I}\right)^{T} \\
& S_{31}^{I I I}=\left(S_{33}^{I I}\right)^{-1} S_{34}^{I I} W\left(S_{44}^{I}-S_{44}^{I I}\right)^{-1} S_{41}^{I}=\left(S_{13}^{I I I}\right)^{T} \\
& S_{32}^{I I I}=\left(S_{33}^{I I}\right)^{-1} S_{34}^{I I} W\left(S_{44}^{I}-S_{44}^{I I}\right)^{-1} S_{42}^{I}=\left(S_{23}^{I I I}\right)^{T} \\
& S_{33}^{I I I}=\left(S_{33}^{I I}\right)^{-1}-\left(S_{43}^{I I}\left(S_{33}^{I I}\right)^{-1}\right)^{T} W\left(S_{44}^{I}-S_{44}^{I I}\right)^{-1} S_{43}^{I I}\left(S_{33}^{I I}\right)^{-1}
\end{aligned}
$$

where

$$
W\left(S_{44}^{I}-S_{44}^{I I}\right)^{-1}=\left[S_{44}^{I}-S_{44}^{I I}+S_{43}^{I I}\left(S_{33}^{I I}\right)^{-1} S_{34}^{I I}\right]^{-1}
$$

For the analysis of SIW corners, one of the branching ports of the SIW T-junction is short-circuited (e.g., port 2 in Figure 3(c)), and the final S-parameters of the SIW corner can be extracted from the S-parameters presented in Equation (18) as:

$$
\begin{aligned}
& {\left[\begin{array}{l}
b_{1} \\
a_{3}
\end{array}\right]=\left[\begin{array}{ll}
S_{11}^{\text {corner }} & S_{12}^{\text {corner }} \\
S_{21}^{\text {corner }} & S_{22}^{\text {corner }}
\end{array}\right]=\left[\begin{array}{l}
a_{1} \\
b_{3}
\end{array}\right]} \\
& S_{11}^{\text {corner }}=S_{11}^{I I I}+S_{12}^{I I I} \cdot\left(-I-S_{22}^{I I I}\right)^{-1} \cdot S_{21}^{I I I} \\
& S_{12}^{\text {corner }}=S_{13}^{I I I}+S_{12}^{I I I} \cdot\left(-I-S_{22}^{I I I}\right)^{-1} \cdot S_{23}^{I I I} \\
& S_{21}^{\text {corner }}=S_{31}^{I I I}+S_{32}^{I I I} \cdot\left(-I-S_{22}^{I I I}\right)^{-1} \cdot S_{21}^{I I I} \\
& S_{22}^{\text {corner }}=S_{33}^{I I I}+S_{32}^{I I I} \cdot\left(-I-S_{22}^{I I I}\right)^{-1} \cdot S_{23}^{I I I}
\end{aligned}
$$

\subsection{Analysis of SIW structures with circular vias}

The MMT analysis presented in the previous sections is applicable to SIW components with square vias. However, in order to analyze SIW structures with circular vias, which are more common in practice, an equivalence between circular vias and square vias has been established [10]. Based on this equivalence, any circular via with diameter of $d$ can be approximated by a square via with side length $l$ as:

$$
l=\left(l_{\text {inner }}+l_{\text {outer }}\right) / 2=d(1 / \sqrt{2}+1) / 2
$$

in which $l_{\text {inner }}$ and $l_{\text {outer }}$ are the side lengths of inscribed and circumscribed squares of the circular via, respectively, as shown in Figure 4.

Based on the MMT analysis presented in this section, the performance of an SIW T-junction with circular vias (Figure 5(a)) is analyzed in Ku-band. 


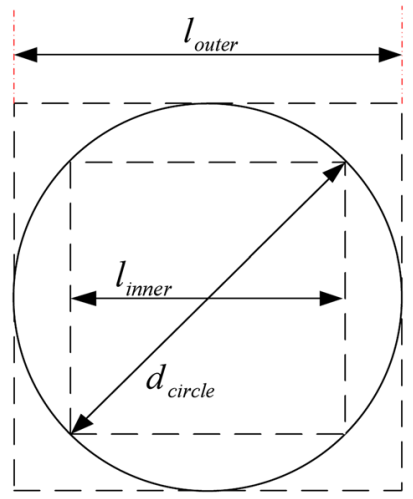

Figure 4. The circle with diameter $d_{\text {circle }}$ and inscribed and circumscribed squares with side lengths $l_{\text {inner }}$ and louter, respectively.

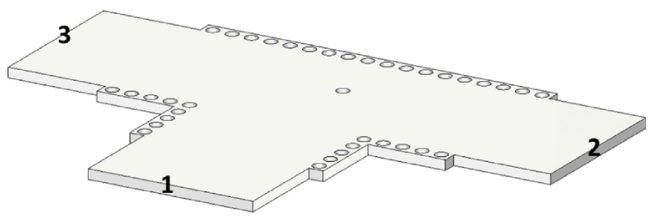

(a)

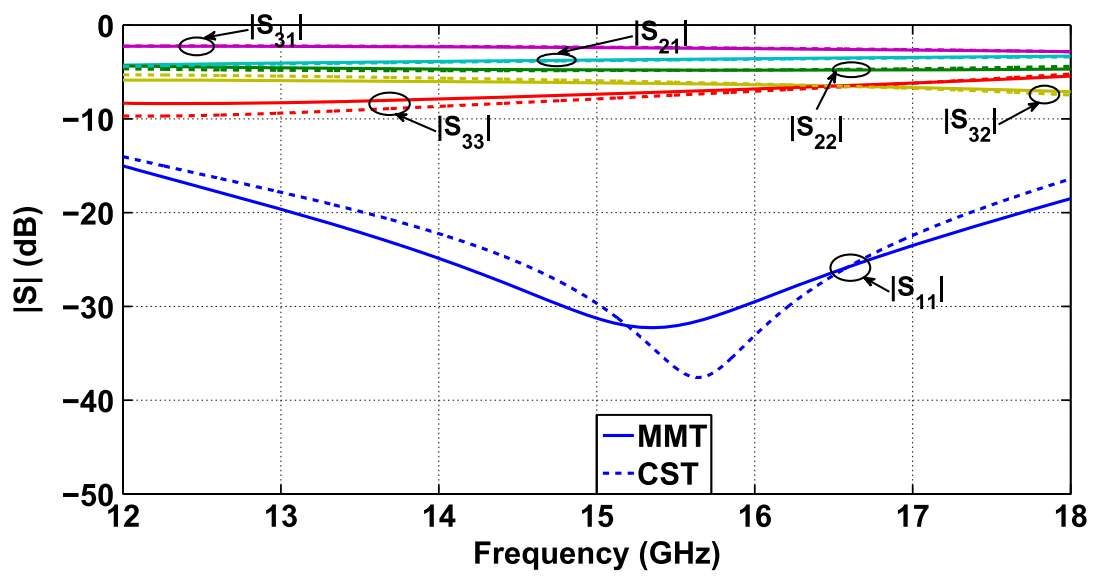

(b)

Figure 5. (a) SIW T-junction with circular vias in Ku-band. The structure is analyzed with MMT (solid line), and the results are compared with CST data (dashed line) (b).

For the design of an SIW component, first, the cutoff frequency $f_{c}$, substrate permittivity $\epsilon_{r}$, and $d / p$ ratio $(0.5<d / p<0.8[12])$ are specified. Once the effective waveguide width of SIW ( $\left.W_{\text {equi }}\right)$ is calculated, the SIW width $\left(a_{S I W}\right)$ can be calculated based on [17]. In this paper, the substrate is chosen as RT/duroid 6002 with $\epsilon_{r}=2.94$ and height $h=0.508 \mathrm{~mm} . f_{c}=10.27 \mathrm{GHz}$ results in $W_{\text {equi }}=8.514 \mathrm{~mm}$, and with $p=1 \mathrm{~mm}$ and $d=0.6444 \mathrm{~mm}$, the SIW width is $a_{S I W}=9 \mathrm{~mm}$. The side length of the square vias in the MMT analysis is $l=0.55 \mathrm{~mm}$. The MMT results for the SIW T-junction with square vias are compared with the simulated data from CST Microwave Studio for the SIW structure with equivalent circular vias (Figure 5(b)). Reasonable agreement is achieved between MMT and CST data, which proves the validity of the proposed analytical approach. 

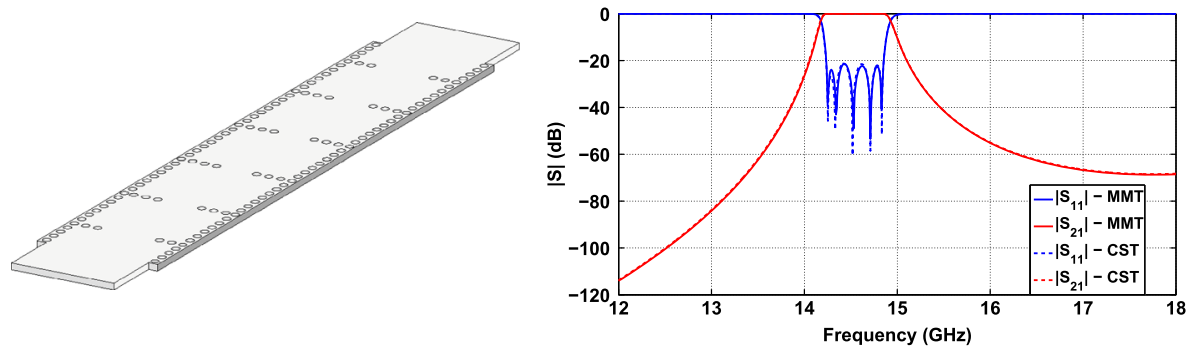

Figure 6. SIW filter for the lower channel of the SIW T-junction diplexer. The structure is analyzed with MMT (solid line), and the results are compared with the CST data (dashed line).
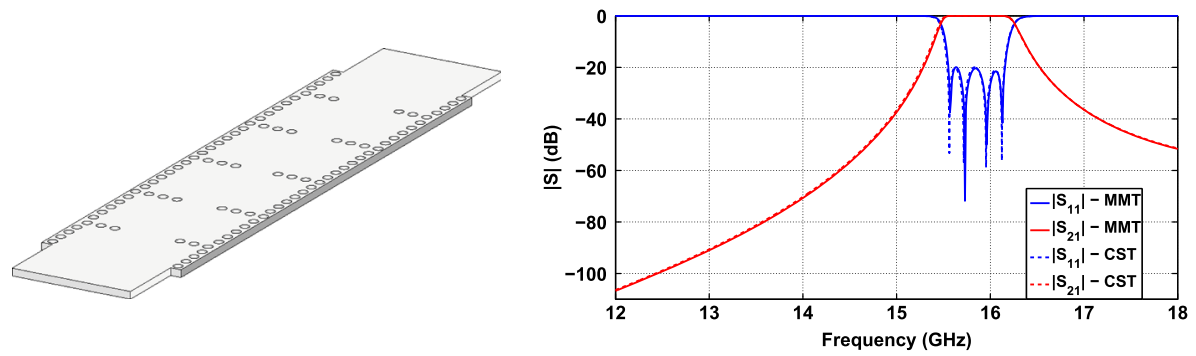

Figure 7. SIW filter for the higher channel of the SIW T-junction diplexer. The structure is analyzed with MMT (solid line), and the results are compared with the CST data (dashed line).

\section{SIW DIPLEXER DESIGN}

The design of the T-junction diplexer starts with the design of two channel filters. The first channel filter with the bandpass located at the lower frequency band is a five-pole filter and has $4.12 \%$ bandwidth at $14.55 \mathrm{GHz}$. This SIW filter is designed and analyzed based on the MMT for SIW structures with square vias, and the analytical data is compared with simulated data with circular vias from CST in Figure 6.

The other filter operating at the higher frequency band is a four-pole filter and has $3.79 \%$ of bandwidth at $15.85 \mathrm{GHz}$. Figure 7 shows the layout of the filter along with the comparison between analytical and simulated data.

Now, the diplexer is formed based on the two presented channel filters. The diplexer is optimized with the analytical approach presented, which saves us significant amount of time, as the presented technique is faster than commercially available field solvers by an order of magnitude [8]. The layout of the final optimized diplexer is presented in Figure 8(a). The designed diplexer is simulated in CST Microwave Studio and also ANSYS HFSS, and the agreement between the analytical and simulated data validates the presented mode-matching based technique for the analysis of SIW components including a T-junction (Figure 8(b)).

\section{SIW CORNER FILTER}

After validation of the presented technique for analyzing SIW T-junctions, one of the branches of the T-junction is being short circuited, and an SIW corner, along with an SIW corner filter, is designed and analyzed.

The SIW corner layout along with its performance is presented in Figure 9. Also, the SIW corner filter, which is a four-pole iris filter, and its S-parameters are presented in Figure 10. Please note that the discrepancy observed between MMT data and simulation data in Figure 10 is because the matrix inversion in Equation (20) limits the number of modes considered in the MMT analysis. Other than that, for both SIW corner and SIW corner filter, the analytical data is in good agreement with the simulated data, which prove the validity of the analytical method.

Copyright $\odot 2014$ John Wiley \& Sons, Ltd 


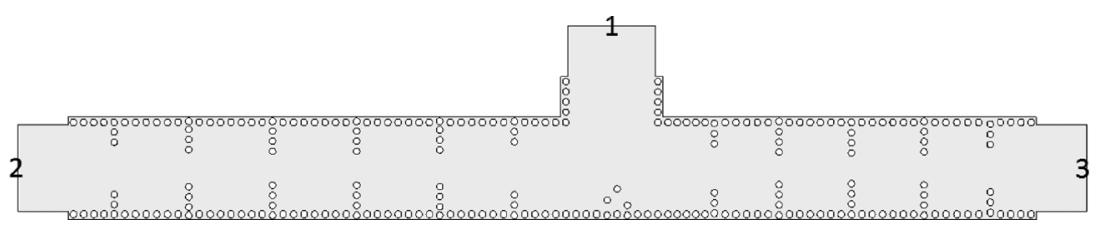

(a)

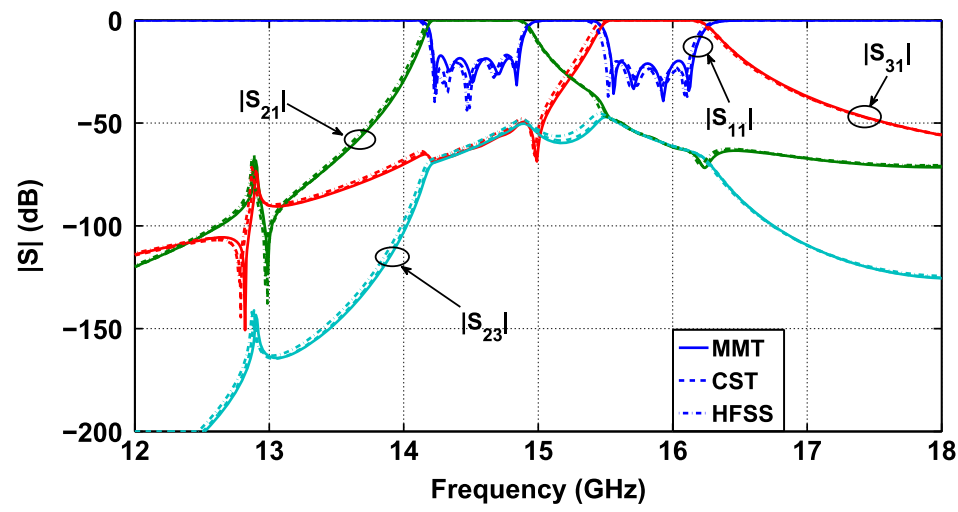

(b)

Figure 8. Diplexer T-junction layout (a), along with S-parameters (b). The structure is analyzed with MMT (solid line), and the data are compared with simulated data from CST (dashed line) and also HFSS (dash-dotted line).
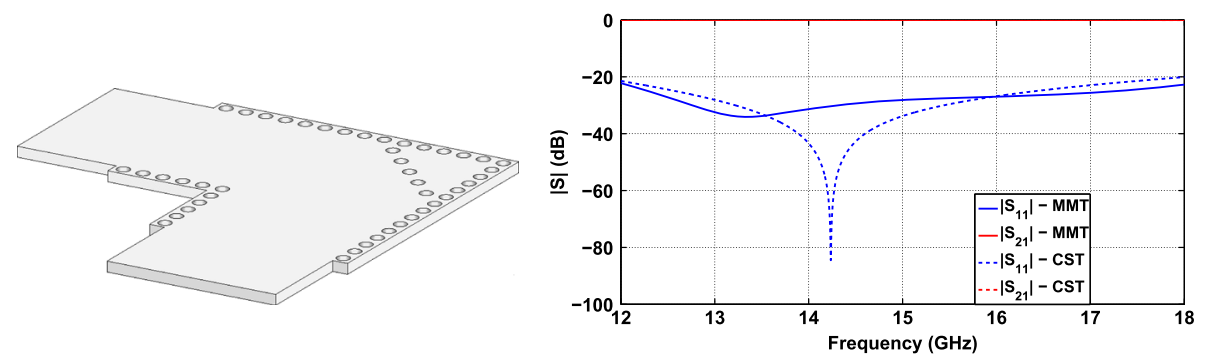

Figure 9. Layout and performance of an SIW corner. MMT data (solid line) have been compared with CST data (dashed line).
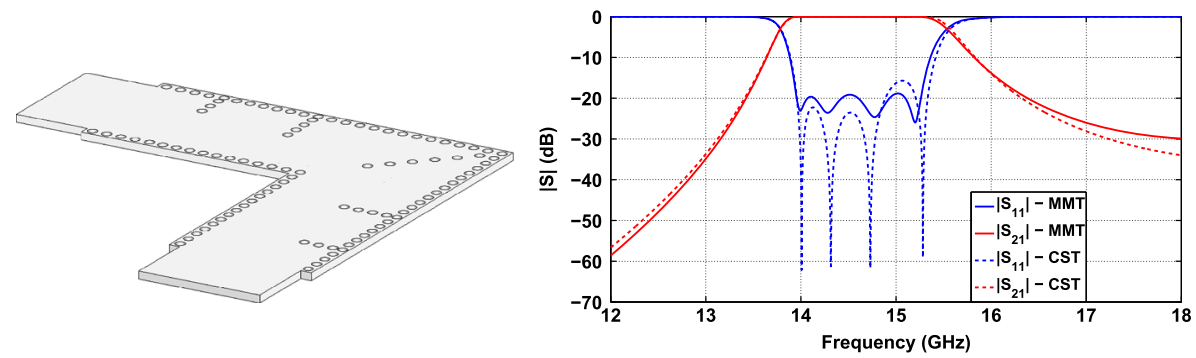

Figure 10. Layout and performance of an SIW corner filter. MMT data (solid line) have been compared with CST data (dashed line). 


\section{CONCLUSION}

The MMT with square vias is a powerful approach for the analysis of SIW components, including filters, T-junctions, and corners. The presented technique is demonstrated by comparing MMT results using square vias with CST and HFSS simulations employing circular vias. Very good agreement is achieved for the T-junction diplexer and the corner filter, thus verifying the MMT-based analysis and design procedure.

\section{ACKNOWLEDGEMENTS}

The authors would like to acknowledge the support of this work from the National Science and Engineering Research Council of Canada (NSERC).

\section{REFERENCES}

1. Chen X, Hong W, Cui T, Wu K. Substrate integrated waveguide (SIW) asymmetric dual-mode filter and diplexer. International Journal of Electronics 2005; 92(12):743-753.

2. Hao ZC, Hong W, Chen JX, Chen XP, Wu K. Planar diplexer for microwave integrated circuits. IEE Proceedings - Microwaves, Antennas and Propagation 2005; 152(6):455-459.

3. Han S, Wang X, Fan Y, Yang Z, He Z. The generalized Chebyshev substrate integrated waveguide diplexer. Progress In Electromagnetics Research 2007; 73:29-38.

4. Tang HJ, Hong W, Chen JX, Luo GQ, Wu K. Development of millimeter-wave planar diplexers based on complementary characters of dual-mode substrate integrated waveguide filters with circular and elliptic cavities. IEEE Transactions on Microwave Theory and Techniques 2007; 55(4):776-782.

5. Hou D, Hong W, Tian L, Liu J, Tang H. A planar triplexer based on substrate integrated waveguide technology for TD-SCDMA applications, Asia-pacific Microwave Conference (APMC) Proceedings, Singapore, December 2009; 2584-2587.

6. Athanasopoulos N, Makris D, Voudouris K. Development of a $60 \mathrm{GHz}$ substrate integrated waveguide planar diplexer, IEEE MTT-S International Microwave Workshop Series on Millimeter
Wave Integration Technologies (IMWS) Proceedings, Sitges, Spain, October 2011; 128-131.

7. Dong Y, Itoh T. Substrate integrated waveguide loaded by complementary split-ring resonators for miniaturized diplexer design. IEEE Microwave and Wireless Components Letters 2011; 21(1): 10-12.

8. Kordiboroujeni Z, Bornemann J. Mode matching design of substrate integrated waveguide diplexers, IEEE MTT-S International Microwave Symposium (IMS) Digest, Seattle, WA, USA, June 2013; 1-3.

9. Bornemann J, Taringou F, Kordiboroujeni Z. A mode-matching approach for the analysis and design of substrate-integrated waveguide components. Frequenz - Journal of RF/Microwave Engr., Photonics and Communications 2011; 65:287-292.

10. Kordiboroujeni Z, Bornemann J, Sieverding T. Mode-matching design of substrate-integrated waveguide couplers, Asia-Pacific Symposium on Electromagnetic Compatibility (APMEC) Proceedings, Singapore, May 2012; 701-704.

11. $\mathrm{Xu} \mathrm{F}, \mathrm{Wu} \mathrm{K}$. Guided-wave and leakage characteristics of substrate integrated waveguide. IEEE Transactions on Microwave Theory and Techniques 2005; 53(1):66-73.

12. Deslandes D, Wu K. Accurate modeling, wave mechanisms, and design considerations of a substrate integrated waveguide. IEEE Transactions on Microwave Theory and Techniques 2006; 54(6):2516-2526

13. Liang XP, Zaki KA, Atia AE. $A$ rigorous three plane modematching technique for characterizing waveguide T-junctions, and its application in multiplexer design. IEEE Transactions on Microwave Theory and Techniques 1991; 39(12):2138-2147.

14. Labay VA, Bornemann J. Generalized modal scattering matrix of discontinuity-distorted waveguide multiport junctions. International Journal of Numerical Modelling: Electronic Networks, Devices and Fields 1997; 10(3): 153-167.

15. Kühn E. A mode-matching method for solving field problems in waveguide and resonator circuits. Archiv fuer Elektronik und Uebertragungstechnik 1973; 27:511-518.

16. Beyer R, Arndt F. The generalized scattering matrix separation technique combined with the MM/FE method for the efficient modal analysis of a comprehensive class of 3D passive waveguide circuits, IEEE MTT-S International Microwave Symposium (IMS) Digest, Orlando, FL , USA, May 1995; 277-280.

17. Kordiboroujeni Z, Bornemann J. Designing the width of substrate integrated waveguide structures. IEEE Microwave and Wireless Components Letters 2013; 23(10): 518-520. 